\title{
Bevacizumab in the therapy for refractory metastatic colorectal cancer
}

\author{
Mary F Mulcahy
}

Northwestern University, Division of Hematology/Oncology, Robert $\mathrm{H}$. Lurie Comprehensive Cancer Center, Chicago IL, USA
Correspondence: Mary F Mulcahy Northwestern University, Division of Hematology/Oncology, Robert H. Lurie Comprehensive Cancer Center, 676 North Saint Clair, Suite 850, Chicago IL 606II, USA

Tel + I 3126954440

Fax + I 3126956189

Email m-mulcahy@northwestern.edu

\begin{abstract}
Colorectal cancer is the second most common cause of cancer related deaths in the United States. Recent developments have led to prolonged survival with the use of sequential lines of chemotherapy agents. The addition of bevacizumab to active chemotherapy has further improved survival when used in the first and second lines of therapy for metastatic colorectal cancer. Evidence supporting the continued use of bevacizumab throughout lines of therapy is accumulating. Clinical trials are underway in which bevacizumab is continued beyond the first line of a chemotherapy and bevacizumab combination regimen. The mechanism by which colorectal cancer may become resistant to bevacizumab is poorly understood. Molecular and biochemical correlates which may identify bevacizumab resistance are an important component in the design of these clinical trials.
\end{abstract}

Keywords: colorectal cancer, bevacizumab, refractory

In the United States population, colorectal cancer (CRC) is the fourth most common cause of cancer and the second most common cause of cancer deaths. In 2007, there are expected to be 153,760 cases of CRC diagnosed, leading to 52,180 deaths (Jemal et al 2007). At the time of presentation, approximately $39 \%$ of patients will have localized disease, $37 \%$ will have regional disease and $19 \%$ will have distant disease; with 5 -year relative survival rates of $90 \%, 68 \%$ and $10 \%$, respectively. Both the incidence and death rate for $\mathrm{CRC}$ has been decreasing over the past 1-2 decades. This decrease can be attributed to improved screening strategies and more active therapy in the adjuvant and advanced disease setting. With current therapy, median overall survival (OS) for patients with advanced CRC approaches two years. Chemotherapy drugs with proven activity in CRC are 5-fluorouracil (5FU), irinotecan (IRI) and oxaliplatin (OX). Monoclonal antibodies targeting the vascular endothelial growth factor (VEGF) and the epidermal growth factor receptor (EGFR) have added to the response rates (RR) and the OS that CRC patients now experience. Chemotherapy may be given as single agents or in combination, with sequential lines of therapy providing improved quality of life, tumor control, and prolonged survival. Enhancing the RR may lead to increasing the number of patients able to undergo potentially curative surgical resection.

\section{Bevacizumab}

Angiogenesis is a required component for normal development as well as tumor growth and metastases. It is a complex multistep process regulated by the balance of endogenous stimulatory and inhibitory factors to maintain equilibrium between vascular growth and cellular demands. During tumorigenesis, solid tumors first undergo an avascular phase of growth until the diffusion of oxygen and the exchange of waste and nutrients become rate limiting. In response to the hypoxic microenvironment, genes which promote angiogenesis are upregulated (Jubb et al 2004). VEGF-A encodes a 
proangiogenic ligand which binds tyrosine kinase receptors on angioblasts and endothelial cells activating the signal transduction cascade resulting in angiogenesis (Ferrara and Davis-Smyth 1997). The expression of VEGF-A is upregulated in a wide range of human tumors and has been associated with a worse prognosis and an increased incidence of disease recurrence (Ellis et al 2000). The vessels formed in the process of tumorigenesis are structurally and functionally abnormal (Jain 1994). An excess of endothelial cells and abnormal perivascular cells contribute to the formation of tortuous, dilated, and saccular blood vessels that are poorly organized and hyperpermeable. The physiologic results are a vascular network that is spatially and temporally heterogeneous, and unable to maintain a gradient between the vascular and interstitial pressures, leading to interstitial hypertension.

Bevacizumab (Avastin ${ }^{\circledR}$; Genentech, Inc., South San Francisco, CA) is a recombinant humanized monoclonal antibody directed against VEGF-A. Preclinical studies demonstrated growth inhibition of human tumor xenografts in response to treatment with VEGF monoclonal antibody (Kim et al 1993). Subsequent clinical trials have established efficacy of bevacizumab in various tumor types, with a survival benefit in the treatment of CRC when combined with chemotherapy. The mechanism of action reflects a restored balance of pro- and anti-angiogenic processes. VEGF inhibition prevents neovascularization and remodels existing vasculature through apoptosis of endothelial cells leading to a decrease in vessel diameter, density and permeability (Jain, 2005). The resultant decrease in interstitial hypertension and normalization of blood vessel structure promotes more efficient delivery of oxygen and therapeutics into the tumor.

Markers demonstrating bevacizumab activity are lacking. Potential biomarkers to predict response to therapy have been explored. Response to therapy has not been associated with mutations in k-ras, b-raf, or p-53 (Ince et al 2005), nor with expression of VEFG-A, thrombospondin-2 or microvessel density (Jubb et al 2006). Phenotypic changes associated with angiogenesis inhibition include changes in vessel structure, vascular permeability, partial pressure of oxygen and interstitial pressure. These changes are not easily identified and surrogate markers have been proposed. A phase I study treated six subjects with rectal cancer with bevacizumab, chemotherapy and radiation (Willett et al 2004). In response to bevacizumab alone, changes in tumor physiology were identified as a decrease in interstitial fluid pressure and, using functional computed tomography, a decrease in tumor blood perfusion and volume. With improved chemotherapeutic regimens for $\mathrm{CRC}$, the addition of bevacizumab has further enhanced RR, progression-free survival (PFS), and OS.

\section{First- and second-line chemotherapy}

The first line of chemotherapy for metastatic CRC is the combination of 5FU, modulated by leucovorin (LV) with IRI (IFL or FOLFIRI) or OX (FOLFOX) for patients with a good performance status. Single agent $5 \mathrm{FU}$, either intravenous or as the oral agent capecitabine, for patients with a compromised performance status is acceptable. This first line of chemotherapy is discontinued when 1) there is evidence of resistance as illustrated by disease progression, 2) there is intolerable cumulative toxicity, or 3) when stable disease is achieved and no further benefit of therapy is evident. When evaluating the benefit of second-line therapy, it is important to distinguish the reason for the discontinuation of the firstline therapy. Metastatic CRC that has demonstrated resistance to first-line therapy may differ biologically from metastatic CRC that was responding or stable on first-line therapy.

A benefit of second-line therapy was first illustrated in a pair of studies comparing IRI with infusion 5FU (Rougier et al 1998) and best supportive care (Cunningham et al 1998) after progression on bolus 5FU. A series of phase II studies performed by Groupe d'Etude ET de Recherche en Cancreologie Onco-Radiotherapic (GERCOR) demonstrated a benefit of $\mathrm{OX}$ when added to $5 \mathrm{FU}$ and $\mathrm{LV}$ after progression on 5FU and LV (Maindrault-Goebel et al 2000; MaindraultGoebel et al 2001). Subsequent studies demonstrated a benefit of combination chemotherapy with either IFL (compared to 5FU/LV and single agent IRI) (Saltz et al 2000), FOLFOX (compared to LV5FU2) (de Gramont et al 2000) or FOLFIRI (compared to 5FU/LV) (Douillard et al 2000) as first-line therapy. Active second-line therapy with FOLFOX was demonstrated after progression on IFL (Rothenberg et al 2003). Further defining the optimal first-line therapy was attempted by the North Central Cancer Treatment Group (comparing IFL with IROX and FOLFOX) (Goldberg et al 2004), and the Gruppo Oncologico Dell'Italia Meridionale (comparing FOLFIRI with FOLFOX) (Colucci et al 2005), among others. While it is clear that infusion schedules of $5 \mathrm{FU}$ are better tolerated than bolus schedules, no clear survival benefit to any first-line regimen emerged as long as all three active drugs are subsequently used (Grothey et al 2004). No optimal sequence was evident in the phase III study comparing FOLFOX followed by FOLIRI or the inverse sequence (Tournigand et al 2004). In all of these studies, second-line 
therapy was defined as therapy used after disease progression on first-line therapy, demonstrating resistance to therapy.

The treatment of metastatic CRC has historically relied on continuous therapy until disease progression. The cumulative neurotoxicity associated with $\mathrm{OX}$ often requires patients to stop while still responding to therapy. This approach confounds the definition of second-line therapy. A stop-andgo strategy was evaluated by the GERCOR in the Optimized 5FU-Oxaliplatin study (OPTIMOX-1) (Tournigand et al 2006). Chemotherapy naïve patients with metastatic CRC were randomly assigned to either FOLFOX-4 administered every 2 weeks until progressive disease or FOLFOX-7 administered every 2 weeks for 6 cycles, then maintenance therapy without oxaliplatin (LV5FU2) for 12 cycles followed by reintroduction of FOLFOX-7. With 620 subjects enrolled, the RR (58.5\% and 59.2\%, respectively), median PFS (9.0 and 8.7 months, respectively) and OS (19.3 and 21.2 months, respectively) were not significantly different in the two groups. The incidence of National Cancer Institute Common Toxicity Criteria (NCI CTC) grade 3 and 4 toxicity was greater for those on continuous oxaliplatin therapy compared to those that stopped after 6 cycles (54.4\% compared to $48.7 \%$, respectively) and specifically grade 3 sensory neurotoxicity (17.9\% and $13.3 \%$ ). This stop-and-go strategy was further evaluated in the OPTIMOX-2 study in which subjects were randomized to 6 cycles of FOLFOX-7 followed by LV5FU2 until progression and reintroduction of FOLFOX-7 (OPTIMOX-1 strategy), or 6 cycles of FOLFOX-7, complete stop of chemotherapy and reintroduction of FOLFOX-7 before the tumor progression reached the baseline measurements (OPTIMOX-2 strategy) (Maindrault-Goebel et al 2007). Originally designed as a phase III study, it was re-designed as a phase II study after accrual suffered due to the demonstrated benefit and availability of bevacizumab. With 202 subjects reported, the median overall survival was 24.6 months in the OPTIMOX-1 arm and 18.9 months in the OPTIMOX-2 $\operatorname{arm}(\mathrm{p}=0.05)$. Those on the OPTIMOX-2 arm had a median chemotherapy free interval of 4 months. Based on these findings, a complete chemotherapy free interval could not be recommended.

\section{Bevacizumab in first-line therapy}

The addition of bevacizumab to the first-line chemotherapy regimen has demonstrated an improvement in outcome parameters. In the pivotal study AVF2107 g, a statistically significant survival benefit was observed when bevacizumab was added to the IFL chemotherapy combination (median OS of 20.3 months compared to 15.6 months, $\mathrm{p}<0.001$ )
(Hurwitz et al 2004). The addition of bevacizumab to 5FU and leucovorin was evaluated in a phase II study, AVF2192 g, for patients thought unfit for IFL (eg, advanced age, compromised performance status, low serum albumin or prior pelvic radiation) (Kabbinavar et al 2005b). The PFS was found to be $67 \%$ improved with the addition of bevacizumab (5.5 vs 9.2 months; $p=0.0002$ ). A pooled analysis of 249 subjects treated with 5FU, LV and bevacizumab from randomized studies further supports the benefit of this combination (median OS for chemotherapy alone and in combination with bevacizumab were 14.6 and 17.9 months, $\mathrm{p}=0.0081$ ) (Kabbinavar et al 2005a). The preliminary results of NO16966 (Cassidy et al 2007; Saltz et al 2007) comparing the infusion schedule of 5 FU and oxaliplatin (FOLFOX) with the oral schedule using capecitabine (XelOx) and a subsequent randomization to bevacizumab or placebo demonstrated superiority with the addition of bevacizumab (median PFS in the chemotherapy plus bevacizumab group 9.4 months compared to 8.0 months in the chemotherapy-alone group, $\mathrm{HR}=0.83, \mathrm{p}=0.0023$ ). This 1.4 month actual benefit is less than has been seen in prior first-line studies and has been attributed to the PFS definition used. Due to changing practice patterns, cumulative chemotherapy toxicities and ongoing studies demonstrating the safety of chemotherapy-free intervals, approximately 3 times as many patients discontinued therapy prior to progression and unrelated to bevacizumab related toxicity in NO16966 than in AVF2107 g.

The OPTIMOX stop-and-go approach including bevacizumab is being pursued in 2 phase III studies. The Spanish Cooperative Group for Gastrointestinal Tumour Therapy is comparing the time to disease progression of combination therapy with capecitabine, oxaliplatin and bevacizumab until disease progression or for 6 cycles followed by bevacizumab alone until disease progression or a premature drop out of the study (Spanish Cooperative Group for Gastrointestinal Tumor Therapy. Study of bevacizumab alone or combined with capecitabine and oxaliplatin as support therapy in metastatic colorectal cancer patients. (Clinical Trials.gov. URL: http:/clinicaltrials.gov/ct/show/NCT00335595). A second trial, the DREAM (Double inhibition, Reintroduction, Erlotinib, Avastin, Metastatic CRC) study is also a phase III trial in which patients receive a regimen of oxaliplatin with either infusion 5FU or capecitabine with bevacizumab (Groupe Cooperateur Multidsciplinaire en Oncologie (GERCOR). Combination chemotherapy and bevacizumab with or without erlotinib in treating patients with metastatic colorectal cancer that cannot be removed by surgery. (URL: http://clinicaltrials. gov/ct/show/NCT00265824). Chemotherapy in all groups 
is stopped after 6 cycles and patients are then randomized to receive either bevacizumab alone or bevacizumab with erlotinib during the chemotherapy-free interval.

\section{Bevacizumab in refractory disease when bevacizumab naïve}

The Eastern Cooperative Oncology Group (ECOG) study 3200 evaluated bevacizumab in second-line therapy for patients that had progressed on therapy with 5FU and IRI (Giantonio et al 2007). The dose of bevacizumab used in this combination was $10 \mathrm{mg} / \mathrm{kg}$ every 2 weeks, as opposed to $5 \mathrm{mg} / \mathrm{kg}$ every 2 weeks as used in the first-line studies. A randomized phase II study in metastatic CRC suggested that a dose of 5 $\mathrm{mg} / \mathrm{kg}$ was more effective than $10 \mathrm{mg} / \mathrm{kg}$; however, the study was not powered to directly compare doses. The choice of this dose was made based on clinical and preclinical data demonstrating a dose-response effect (Manley et al 2002; Yang et al 2003). A total of 829 subjects were randomized to a regimen of FOLFOX-4, FOLFOX-4 with bevacizumab or bevacizumab alone after documented progression on $5 \mathrm{FU}$ and IRI. The primary endpoint was OS with a $95 \%$ power to detect a difference of $50 \%$. The single agent bevacizumab arm was closed to accrual after a planned interim analysis suggested an inferior survival compared to the two chemotherapy containing arms. With a median follow up of 28 months, the median OS for FOLFOX-4 with bevacizumab, FOLFOX-4, and bevacizumab alone was 12.9, 10.8, and 10.2 months, respectively with a hazard ratio of $0.75(p=0.0011)$ for the chemotherapy containing arms. The median PFS demonstrated a benefit for the FOLFOX-4 with bevacizumab arm compared to the FOLFOX-4 arm (7.3 compared to 4.7 months, hazard ratio for progression $=0.61 ; \mathrm{p}<.0001)$. The median duration of therapy was 10 cycles for those treated with the FOLFOX-4 and bevacizumab, 7 for those with FOLFOX-4, and 4 for those with bevacizumab alone. The toxicity profile reflects the longer duration of therapy for the FOLFOX-4 with bevacizumab regimen. There were higher rates of NCI CTC grade $3 / 4$ neuropathy $(16 \% / 0.3 \%$ compared to $8.8 \% / 0.4 \%)$, hypertension (5.2\%/1.0\% compared to $1.4 \% / 0.4 \%)$, bleeding (3.1\%/0.3\% compared to $0.4 \% / 0)$ and vomiting $(8.7 \% / 1.4 \%$ compared to $2.8 \% / 0.4 \%$ ). The toxicity profile was comparable to that seen in studies using a bevacizumab dose of $5 \mathrm{mg} / \mathrm{kg}$. This study not only demonstrated activity of bevacizumab in previously treated patients, but confirmed its activity with FOLFOX therapy.

The treatment of multidrug refractory disease was addressed in the National Cancer Institute Treatment Referral Centre trial, TRC-0301 (Chen et al 2006). Patients with progressive CRC despite treatment with the active agents 5FU, OX, and IRI were treated with 5FU (bolus or infusion) in combination with bevacizumab. Disease control, defined as the combination of partial response and stable disease, was achieved in $33 \%$ of the subjects and PFS was found to be 3.5 months (95\% CI of 2.0-4.3 months). The specified primary outcome in this trial was objective RR which was $1 \%$ in this heavily pre-treated group of patients.

Cetuximab is a monoclonal antibody inhibiting the EGFR. For patients with irinotecan refractory disease, cetuximab alone or in combination with irinotecan demonstrated a RR of $10.8 \%$ and $22.9 \%$ ( $p<0.074)$ respectively, a time to treatment failure of 1.5 months and 4.1 months $(\mathrm{p}<0.001)$ respectively, but no significant improvement in median OS (Cunningham et al 2004). A subsequent study added bevacizumab to both arms, combining bevacizumab with cetuximab alone or with cetuximab and irinotecan, for irinotecan refractory disease (Saltz et al 2005). This study was designed as a phase III study for patients that had not received prior anti-EGFR or anti-VEGF therapy, but suffered from poor accrual with the FDA approval of bevacizumab for first-line therapy and cetuximab for refractory disease. It was redesigned as a randomized phase II study with the primary objective of evaluating the safety and efficacy of concurrent administration of cetuximab and bevacizumab with or without irinotecan. Eighty-one subjects were enrolled; all had demonstrated tumor growth on an irinotecan containing regimen during any line of therapy; demonstration of EGFR expression was not required for enrollment. Subjects had received between 1 and 8 prior chemotherapy regimens with a median of three and most had received prior oxaliplatin. In this heavily pretreated population, the RR and time to progression (TTP) for patients receiving cetuximab and bevacizumab was $20 \%$ and 5.6 months respectively, and for those receiving irinotecan, cetuximab and bevacizumab was $37 \%$ and 7.9 months, respectively. As compared to the historical controls, the addition of bevacizumab in refractory disease enhanced the effects of cetuximab alone (RR 20\% and 11\%, TTP 5.6 months and 1.5 months; respectively) and the combination of irinotecan and cetuximab (RR 37\% and 23\%, TTP 7.9 months and 4 months; respectively).

\section{Continued bevacizumab in refractory disease}

As of yet, there is no measurable marker to indicate resistance to bevacizumab. Bevacizumab acts at the level of the endothelial cell, which unlike the tumor should be a genetically stable unit. Induced anti-VEGF inhibitor antibodies 


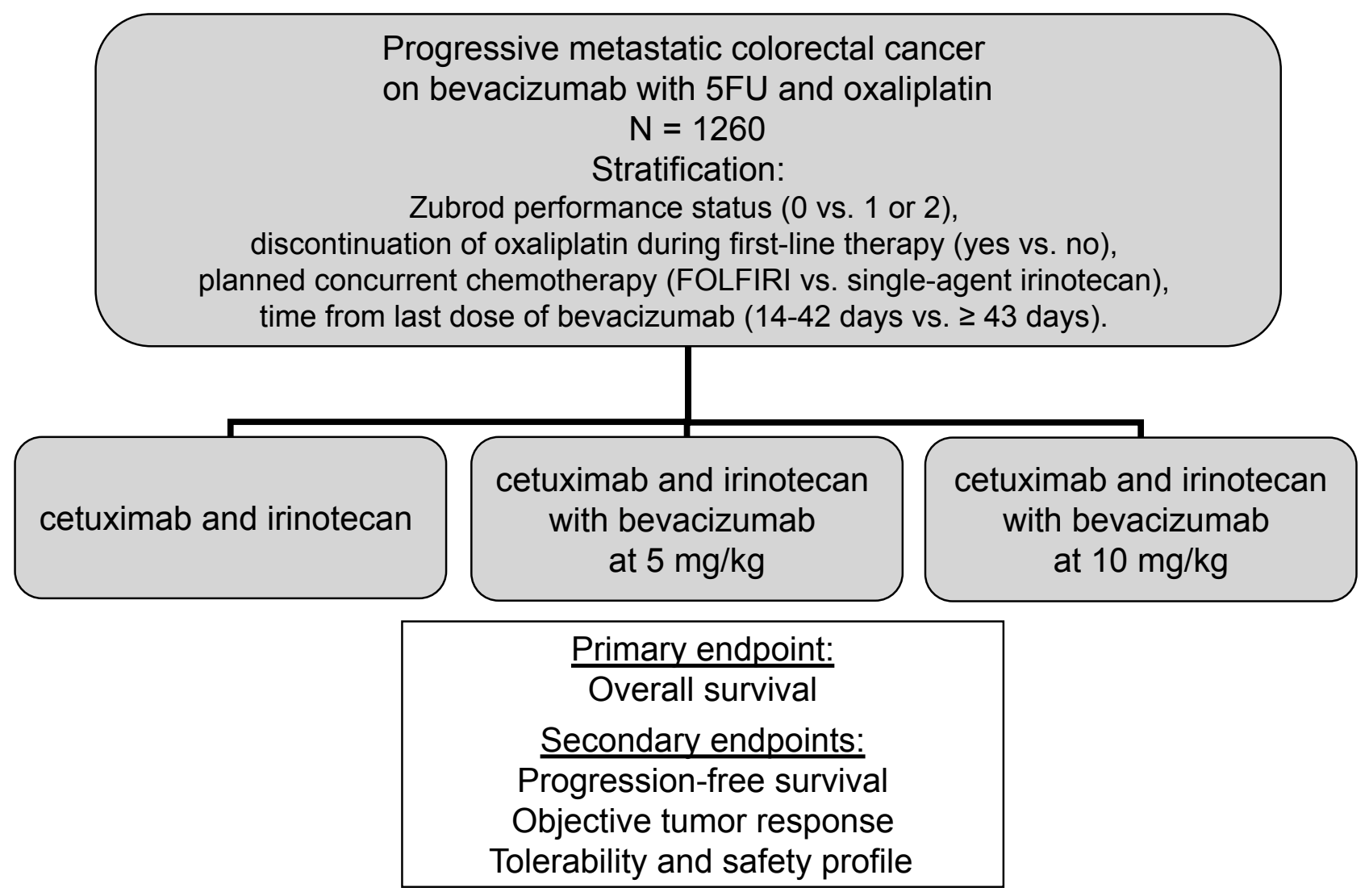

Figure I S0600/iBET schema: irinotecan and cetuximab with or without bevacizumab in treating patients with metastatic colorectal cancer that progressed during first-line therapy.

have not been demonstrated. Resistance to bevacizumab activity may be acquired by upregulating angiogenesis activators or bypassing VEGF pathways. There are no evidence-based studies to support the use of bevacizumab beyond progression when used in the first line of therapy. Extrapolation of subgroups from randomized studies, anecdotal reports and observational studies provide the only evidence of activity of bevacizumab in refractory disease while on a bevacizumab containing regimen (Hurwitz et al 2006).

The BRiTE registry (Bevacizumab Regimens: Investigation of Treatment Effects and safety) was initiated in 2004 to evaluate the safety and efficacy of bevacizumab in combination with chemotherapy in the first line of therapy in a large, less-selected community based population. Using this prospectively collected database, the evaluation of bevacizumab when used after progression on a bevacizumab containing regimen provides important efficacy and safety information (Grothey et al 2007). Data were collected at baseline and every 3 months, specifically pertaining to changes in or discontinuation of therapy. Bevacizumab beyond progression (BBP) was defined as exposure to bevacizumab more than
28 days after first progression on a bevacizumab containing regimen. Patients who had progressed on first-line therapy were grouped into 1) no subsequent therapy, 2) post progressive therapy without bevacizumab (no BBP), or 3 ) post progressive therapy with bevacizumab (BBP). Of 1445 patients with progressive disease, $642(44 \%)$ received BBP, 531 (37\%) received no BBP, and 253 (18\%) received no treatment beyond first progression. The OS from the time of progression was 19.2 months (95\% CI of 16.8-20.7 months), 9.5 months (95\% CI of 8.4-11.2 months) and 3.6 months ( $95 \%$ CI of 2.7-4.3), in each group respectively. In a multivariate analysis of independent effects of patient-related factors on survival, the hazard ratio for the use of BBP compared to no BBP was 0.46 (95\% CI 0.41-0.57). While there are many shortcomings of an observation study such as this, these findings do support the evaluation of BBP in the prospective randomized phase III intergroup trial S0600/iBET (Figure 1) (Southwest Oncology Group. Irinotecan and cetuximab with or without bevacizumab in treating patients with metastatic colorectal cancer that progressed during first-line therapy. (Clinical Trials.gov. URL: http://www.clinicaltrials.gov/ct/ show/NCT00499369). 


\section{Conclusion}

Most patients that do not have a contraindication to receiving bevacizumab are receiving it in the first line of therapy. Bevacizumab has been shown in randomized studies to enhance the effects of chemotherapy regardless of the chemotherapy regimen used, and in the first line and second line of active therapy. Bevacizumab demonstrated no significant activity as a single agent in ECOG 3200 (Giantonio et al 2007) nor when combined with 5FU in 5FU refractory disease in TRC-0301 (Chen et al 2006). Bevacizumab did demonstrate activity when used in multiagent chemotherapy refractory disease in combination with EGFR inhibition and through subsequent lines of active chemotherapy regimens as in the BRiTE registry.

Appropriately powered clinical trials are needed to identify the role of bevacizumab through multiple lines of chemotherapy regimens. Study designs need to include 1) a predetermined definition of 'lines of therapy', 2) an adequate sample size to allow for drop-out as subjects progress through lines of therapy, 3) appropriate independent review and 4) correlative studies to identify molecular changes which may account for the findings. Studies are ongoing in which bevacizumab is being evaluated during elective chemotherapy-free intervals for patients maintaining disease control, and with a second-line regimen of therapy for patients progressing on first-line therapy. Until the results of these studies are known, the use bevacizumab in refractory disease is supported by a high level of evidence in bevacizumab-naïve patients, but a lower level of evidence in bevacizumab-treated patients.

\section{References}

Cassidy JCS, Diaz-Rubio E, Scheithauer W, et al. 2007. XELOX vs. FOLFOX4: Efficacy results from XELOX-1/NO16966, a randomized phase III trial in first-line metastatic colorectal cancer (MCRC). In Grunberg SM. (Ed.) 2007, Gastrointestinal Cancers Symposium, Orlando Florida, American Society of Clinical Oncology.

Chen HX, Mooney M, Boron M, et al. 2006. Phase II multicenter trial of bevacizumab plus fluorouracil and leucovorin in patients with advanced refractory colorectal cancer: an NCI Treatment Referral Center Trial TRC-0301. J Clin Oncol, 24:3354-60.

Colucci G, Gebbia V, Paoletti G, et al. 2005. Phase III randomized trial of FOLFIRI versus FOLFOX4 in the treatment of advanced colorectal cancer: a multicenter study of the Gruppo Oncologico Dell'Italia Meridionale. J Clin Oncol, 23:4866-75.

Cunningham D, Humblet Y, Siena S, et al. 2004. Cetuximab monotherapy and cetuximab plus irinotecan in irinotecan-refractory metastatic colorectal cancer. $N$ Engl J Med, 351, 337-45.

Cunningham D, Pyrhonen S, James RD, et al. 1998. Randomised trial of irinotecan plus supportive care versus supportive care alone after fluorouracil failure for patients with metastatic colorectal cancer. Lancet, $352: 1413-8$

De Gramont A, Figer A, Seymour M, et al. 2000. Leucovorin and fluorouracil with or without oxaliplatin as first-line treatment in advanced colorectal cancer. J Clin Oncol, 18:2938-47.
Douillard JY, Cunningham D, Roth AD, et al. 2000. Irinotecan combined with fluorouracil compared with fluorouracil alone as first-line treatment for metastatic colorectal cancer: a multicentre randomised trial. Lancet, 355:1041-7.

Ellis LM, Takahashi Y, Liu W, et al. 2000. Vascular endothelial growth factor in human colon cancer: biology and therapeutic implications. Oncologist, 5(Suppl 1):11-5.

Ferrara N, Davis-Smyth T. 1997. The biology of vascular endothelial growth factor. Endocr Rev, 18:4-25.

Giantonio BJ, Catalano PJ, Meropol NJ, et al. 2007. Bevacizumab in combination with oxaliplatin, fluorouracil, and leucovorin (FOLFOX4) for previously treated metastatic colorectal cancer: results from the Eastern Cooperative Oncology Group Study E3200. J Clin Oncol, 251539-44.

Goldberg RM, Sargent DJ, Morton RF, et al. 2004. A randomized controlled trial of fluorouracil plus leucovorin, irinotecan, and oxaliplatin combinations in patients with previously untreated metastatic colorectal cancer. J Clin Oncol, 22:23-30.

Grothey A, Sargent D, Goldberg RM, et al. 2004. Survival of patients with advanced colorectal cancer improves with the availability of fluorouracil-leucovorin, irinotecan, and oxaliplatin in the course of treatment. J Clin Oncol, 22:1209-14.

Grothey A, Sugrue M, Hedrick E, et al. and The Brite Study Investigators. 2007. Association between exposure to bevacizumab (BV. beyond first progression (BBP. and overall survival (OS. in patients (pts. with metastatic colorectal cancer (mCRC.: Results from a large observational study (BRiTE. J Clin Oncol 2007 ASCO Annual Meeting Proceedings, 25.

Hurwitz H, Fehrenbacher L, Novotny W, et al. 2004. Bevacizumab plus irinotecan, fluorouracil, and leucovorin for metastatic colorectal cancer. N Engl J Med, 350:2335-42.

Hurwitz HI, Honeycutt W, Haley S, et al. 2006. Long-term treatment with bevacizumab for patients with metastatic colorectal cancer: case report. Clin Colorectal Cancer, 6:66-9.

Ince WL, Jubb AM, Holden SN, et al. 2005. Association of k-ras, b-raf, and $\mathrm{p} 53$ status with the treatment effect of bevacizumab. J Natl Cancer Inst, 97:981-9.

Jain RK. 1994. Barriers to drug delivery in solid tumors. Sci Am, 271:58-65.

Jain RK. 2005. Normalization of tumor vasculature: an emerging concept in antiangiogenic therapy. Science, 307:58-62.

Jemal A, Siegel R, Ward E, et al. 2007. Cancer statistics, 2007. CA Cancer J Clin, 57:43-66.

Jubb AM, Hurwitz HI, Bai W, et al. 2006. Impact of vascular endothelial growth factor-A expression, thrombospondin-2 expression, and microvessel density on the treatment effect of bevacizumab in metastatic colorectal cancer. J Clin Oncol, 24:217-27.

Jubb AM, Pham TQ, Hanby AM, et al. 2004. Expression of vascular endothelial growth factor, hypoxia inducible factor 1alpha, and carbonic anhydrase IX in human tumours. J Clin Pathol, 57:504-12.

Kabbinavar FF, Hambleton J, Mass RD, et al. 2005a. Combined analysis of efficacy: the addition of bevacizumab to fluorouracil/leucovorin improves survival for patients with metastatic colorectal cancer. J Clin Oncol, 23:3706-12.

Kabbinavar FF, Schulz J, McCleod M, et al. 2005b. Addition of bevacizumab to bolus fluorouracil and leucovorin in first-line metastatic colorectal cancer: results of a randomized phase II trial. J Clin Oncol, 23:3697-705

Kim KJ, Li B, Winer J, et al. 1993. Inhibition of vascular endothelial growth factor-induced angiogenesis suppresses tumour growth in vivo. Nature, 362:841-4.

Maindrault-Goebel F, De Gramont A, Louvet C, et al. 2000. Evaluation of oxaliplatin dose intensity in bimonthly leucovorin and 48-hour 5-fluorouracil continuous infusion regimens (FOLFOX. in pretreated metastatic colorectal cancer. Oncology Multidisciplinary Research Group (GERCOR). Ann Oncol, 11:1477-83. 
Maindrault-Goebel F, De Gramont A, Louvet C, et al. 2001. High-dose intensity oxaliplatin added to the simplified bimonthly leucovorin and 5 -fluorouracil regimen as second-line therapy for metastatic colorectal cancer (FOLFOX 7). Eur J Cancer, 37:1000-5.

Maindrault-Goebel F, Lledo G, Chibaudel B, et al. GERCOR. 2007. Final results of OPTIMOX2, a large randomized phase II study of maintenance therapy or chemotherapy-free intervals (CFI) after FOLFOX in patients with metastatic colorectal cancer (MRC): A GERCOR study. J Clin Oncol, 2007 ASCO Annual Meeting Proceedings, 25.

Manley PW, Martiny-Baron G, Schlaeppi JM, et al. 2002. Therapies directed at vascular endothelial growth factor. Expert Opin Investig Drugs, 11:1715-36.

Rothenberg ML, Oza AM, Bigelow RH, et al. 2003. Superiority of oxaliplatin and fluorouracil-leucovorin compared with either therapy alone in patients with progressive colorectal cancer after irinotecan and fluorouracil-leucovorin: interim results of a phase III trial. $J$ Clin Oncol, 21:2059-69.

Rougier P, Van Cutsem E, Bajetta E, et al. 1998. Randomised trial of irinotecan versus fluorouracil by continuous infusion after fluorouracil failure in patients with metastatic colorectal cancer. Lancet, 352:1407-12.

Saltz LB, Cox JV, Blanke C, et al. 2000. Irinotecan plus fluorouracil and leucovorin for metastatic colorectal cancer. Irinotecan Study Group. N Engl J Med, 343:905-14.
Saltz LB, Diaz-Rubio E, Scheithauer W, et al. 2007. Bevacizumab (Bev in combination with XELOX or FOLFOX4: Efficacy results from XELOX-1/NO16966, a randomized phase III trial in the first-line treatment of metastatic colorectal cancer (MCRC). In Grunberg SM. (Ed). 2007 Gastrointestinal Cancers Symposium, Orlando Florida, American Society of Clinical Oncology.

Saltz LB, Hochster H, Wadler S, et al. 2005. Randomized phase II Trial of cetuximab/bevacizumab/irinotecan (CBI) versus cetuximab/bevacizumab (CB) in irinotecan-refractory colorectal cancer. J Clin Oncol, 2005 ASCO Annual Meeting Proceedings, 23.

Tournigand C, Andre T, Achille E, et al. 2004. FOLFIRI followed by FOLFOX6 or the reverse sequence in advanced colorectal cancer: a randomized GERCOR study. J Clin Oncol, 22:229-37.

Tournigand C, Cervantes A, Figer A, et al. 2006. OPTIMOX1: a randomized study of FOLFOX4 or FOLFOX7 with oxaliplatin in a stop-andGo fashion in advanced colorectal cancer - a GERCOR study. $J$ Clin Oncol, 24:394-400.

Willett CG, Boucher Y, Di Tomaso E, et al. 2004. Direct evidence that the VEGF-specific antibody bevacizumab has antivascular effects in human rectal cancer. Nat Med, 10:145-7.

Yang JC, Haworth L, Sherry RM, et al. 2003. A randomized trial of bevacizumab, an anti-vascular endothelial growth factor antibody, for metastatic renal cancer. $N$ Engl J Med, 349:427-34 
